\title{
Thermodynamical fluctuations and photo-thermal shot noise in gravitational wave antennae
}

\author{
V. B. Braginsky, M.L. Gorodetsky and S. P. Vyatchanin \\ Physics Faculty, Moscow State University, \\ Moscow 119899, Russia \\ e-mail:brag@hbar.phys.msu.su
}

\begin{abstract}
Thermodynamical fluctuations of temperature in mirrors of gravitational wave antennae are transformed through thermal expansion coefficient into additional noise. This source of noise, which may also be interpreted as fluctuations due to thermoelastic damping, may not be neglected and leads to the necessity to reexamine the choice of materials for the mirrors. Additional source of noise are fluctuations of the mirrors' surfaces caused by optical power absorbed in dielectrical reflective layers.
\end{abstract}

\section{INTRODUCTION}

The full scale terrestrial gravitational wave antennae are now in the process of assembling and tuning. It is expected that in the year 2001 in one of these antennae (project LIGOI) the sensitivity in units of amplitude of the perturbation of metric will be at the level $h \simeq 1 \times 10^{-21}$ [1],2]. This value means that the optical readout system will be able to register small displacement between two test masses (two heavy optical mirrors placed in vacuum pipe at the distance $L=4 \times 10^{5} \mathrm{~cm}$ ) with the resolution $\Delta L_{\text {grav }} \simeq \frac{1}{2} h L \simeq 2 \times 10^{-16} \mathrm{~cm}$. This displacement may be produced by a burst of gravitational wave generated in astrophysical catastrophe. The characteristic frequency in this wave packet and, correspondingly, in the 
displacement $\omega_{\text {grav }}$ has to be of the order of $2 \pi \times 10^{2} \mathrm{~s}^{-1}$. The improvement of the readout system and better isolation of the mirrors from different sources of noise will permit to achieve higher sensitivity: in the year 2005 the planned level of sensitivity has to be better than $h \simeq 2 \times 10^{-23}$ and correspondingly it will be necessary to register $\Delta L_{\text {grav }} \leq 4 \times 10^{-18} \mathrm{~cm}$. It is worth noting that in the prototype LIGO (where $L=4 \times 10^{3} \mathrm{~cm}$ ) the already achieved resolution was $\Delta L_{\text {grav }}=3 \times 10^{-16} \mathrm{~cm} \mathrm{[2].}$

The important feature of this antenna is that the optical readout system does not register displacements between the centers of the mirrors' masses but between the internal surfaces of the mirrors. These surfaces are coated by several thin dielectrical layers, which provide high optical reflectivity of the mirrors and high sensitivity to the displacements. The necessity to measure the displacements between the internal surfaces inevitably determines the requirement for high quality factors $Q_{\text {int }}$ (small loss angle) of internal mechanical modes of the mirrors, which provide relatively small values of random thermal fluctuations of the mirrors related to the mirrors' center of mass because the value of $\omega_{\text {grav }}$ is substantially smaller than the eigen-frequencies of internal modes. We shall call as Brownian the fluctuations which are usually calculated from phenomenological model of structural losses in the material in order to distinguish them from another type of thermodynamical fluctuations which we describe below. This requirement for high $Q_{i n t}$ determines the choice of the mirrors' material. On the first stage of LIGO the mirrors are made of very pure fused silica $\left(\mathrm{SiO}_{2}\right)$, because the values of $Q_{\mathrm{int}_{\mathrm{SiO}}}$ exceed $2 \times 10^{7}$ [3, 田.

At present the possibility to make the mirrors for the second stage of LIGO from sapphire monocrystal $\left(\mathrm{Al}_{2} \mathrm{O}_{3}\right)$ is widely debated, because more than 20 years ago the achieved value of $Q_{\mathrm{int} \mathrm{Al}_{2} \mathrm{O}_{3}}$ for this material was $3 \times 10^{8}$ [0] .

We show below that the value of $Q_{\text {int }}$ is not the only parameter which determines the choice of material from which the mirror has to be manufactured. Till now the experimentalists' interest has neen concentrated on the reduction of the Brownian motion of the mirrors' surfaces (as it was described above), to the reduction of thermal fluctuations of the centers of mirrors' masses due to the friction in the mirrors' suspension [6.7] and to excess noise in 
the suspensions [9]. To our best knowledge nobody has so far paid attention to nonlinear effects which may be "responsible" for additional fluctuations of the mirrors surface, which may exceed traditional Brownian noise for low frequencies. The simplest of these effects is thermal expansion, originated from anharmonisity of interaction potential of atoms in solid state. The following numerical example illustrates the role of nonzero value of thermal expansion coefficient $\alpha$ of the mirrors material. Suppose that the surface layer of the mirror having thickness $l_{T} \simeq 10^{-2} \mathrm{~cm}$ (it is the characteristic length of the decay of Fourier thermal wave at frequency $2 \pi \times 10^{2} \mathrm{~s}^{-1}$ in fused silica) is heated due to some process on $\delta T$. Then the distance between the surface of the mirror and its center of mass will also change on the value of $\delta l$ :

$$
\delta l=\alpha l \delta T \simeq 1 \times 10^{-17} \mathrm{~cm} \times\left(\frac{\alpha}{5 \times 10^{-7} \mathrm{~K}^{-1}}\right) \times\left(\frac{l_{T}}{1 \times 10^{-2} \mathrm{~cm}}\right) \times\left(\frac{\delta T}{2 \times 10^{-9} \mathrm{~K}}\right) .
$$

The estimate (11) shows, that if the mirrors are made of fused silica and if the temperature of the first layer $\left(10^{-2} \mathrm{~cm}\right.$ thick) of the mirror changes by $2 \mathrm{nK}$ during $5 \times 10^{-3} \mathrm{~s}$ then the antenna will register a "burst" of gravitational radiation with amplitude of the order of $h \simeq 10^{-22}$ with $\omega_{\text {grav }}=2 \pi \times 10^{2} \mathrm{~s}$.

The aim of this article is to present the results of the analysis of the two effects which are "responsible" for random fluctuations of temperature in the bulk of the mirrors and which may mimic at certain level the gravitational wave if $\alpha \neq 0$ : photo-thermal shot noise (due to random absorption of optical photons in the surface layer of the mirror) and thermodynamical fluctuations of temperature.

As in both effects we are interested in the fluctuations of the coordinate averaged over the spot on mirror surface with radius $r_{0} \simeq 1.5 \mathrm{~cm}$ of laser beam, which is much smaller than the radius of mirror $\simeq 15 \mathrm{~cm}$, we replace the mirror by half-space: $0 \leq x<\infty, \infty<$ $y<\infty, \infty<z<\infty$. We consider also for simplicity this half-space to be isotropic (the anisotropy of different constants for sapphire is not very large). The role of these two effects for the condition to reach the standard quantum limit of frequency stability was analyzed more than 20 years ago 10 . 
As at room temperature the power radiated from the surface (Stephan-Boltzmann law) is much lower than the heat exchange due to thermal conductivity (Fourier law), below we use the simplified boundary condition:

$$
\left.\frac{\partial u(x, y, z, t)}{\partial x}\right|_{x=0}=0,
$$

where $u$ is the deviation of temperature from the mean value $T$.

For the calculations we use approximation in which we neglect the effects of limited speed of sound but take into account thermal relaxation.

\section{PHOTO-THERMAL SHOT NOISE IN THE MIRRORS OF THE ANTENNAE}

The multilayer coating of the mirrors absorbs a fraction of power $W$ of the optical beam, circulating between the mirrors of Fabry-Perot resonator. This fraction for the best existing today coatings has not been measured with good precision yet, but for estimates the usually accepted value of this fraction is approximately equal to $5 \times 10^{-7} \div 10^{-6}$ [11]. The value of $W$ sufficient to reach $h \simeq 10^{-22}$ (which is close to Standard Quantum Limit (SQL) of sensitivity) is approximately $W \simeq 1 \times 10^{6} \mathrm{Watt}=10^{13} \mathrm{erg} / \mathrm{sec}$ or $\dot{N}_{0} \simeq 5 \times 10^{24} \mathrm{photon} / \mathrm{s}$ [12]. It is reasonable to estimate that each mirror absorbs $W_{a b s} \simeq 1$ Watt $=10^{7} \mathrm{erg} / \mathrm{s}$ or $\dot{N} \simeq 5 \times 10^{18}$ photon/s. Each absorbed optical photon having energy $\hbar \omega_{0} \simeq 2 \times 10^{-12} \mathrm{erg}$ gives birth to a bunch of approximately 50 thermal phonons. Each of these phonons has a relatively short free path $l^{*}$ (for $\mathrm{SiO}_{2}$ and $\mathrm{Al}_{2} \mathrm{O}_{3}$ the values are $l_{\mathrm{SiO}_{2}}^{*} \simeq 8 \times 10^{-8} \mathrm{~cm}$ and $l_{\mathrm{Al}_{2} \mathrm{O}_{3}}^{*} \simeq 5 \times 10^{-7} \mathrm{~cm} \mathrm{[13]).} \mathrm{After} \mathrm{a} \mathrm{very} \mathrm{short} \mathrm{time} \mathrm{interval} \mathrm{which} \mathrm{is} \mathrm{a} \mathrm{few} \mathrm{times} \mathrm{longer} \mathrm{than}$ $l^{*} / v_{s} \simeq 10^{-12} \mathrm{~s}$ (where $v_{s}$ is the speed of sound) these phonons will produce a local jump of temperature inside the coating $(5 \div 10) \times 10^{-4} \mathrm{~cm}$ thick. As we want to know the variance of temperature during the averaging time $\pi / \omega_{\text {grav }} \sim 5 \times 10^{-3} \mathrm{~s}$ which is several orders longer than $l^{*} / v_{s}$ and as during this time a large number $\left(\dot{N} \pi / \omega_{\text {grav }} \sim 2.5 \times 10^{16}\right)$ of photons are creating bunches of thermal phonons - it is reasonable to use the model of shot noise. As the length $l_{T}$ is much smaller than the radius of laser beam $r_{0} \simeq 1.5 \mathrm{~cm}$ for semi-qualitative analysis one can use one dimensional model, described by thermal conductivity equation: 


$$
\begin{aligned}
\frac{\partial u(x, t)}{\partial t}-a^{2} \frac{\partial^{2} u(x, t)}{\partial x^{2}} & =\frac{w}{\rho C \pi r_{0}^{2}} 2 \delta(x), \\
\left.\frac{\partial u(x, t)}{\partial x}\right|_{x=0} & =0, \quad 0 \leq x<\infty, \\
\left\langle w(t) w\left(t^{\prime}\right)\right\rangle & =\hbar \omega_{0} W_{0} \delta\left(t-t^{\prime}\right), \quad w(t)=W_{\text {abs }}-W_{0}, \quad W_{0}=\left\langle W_{a b s}(t)\right\rangle,
\end{aligned}
$$

where $a^{2}=\lambda^{*} /(\rho C), \lambda^{*}$ is thermal conductivity, $\rho$ is density and $C$ is specific heat capacity.

Assuming that displacement $X_{1 \mathrm{D}}$ (which is of interest) is proportional to temperature $u$ averaged along the axis $x$

$$
X_{1 \mathrm{D}}=\alpha \int_{0}^{\infty} d x u(x, t)
$$

it is not difficult to obtain the spectral density of $X_{1 \mathrm{D}}$ t:

$$
S_{\mathrm{TS} 1 \mathrm{D}}(\omega)=2 \alpha^{2} \frac{\hbar \omega_{0} W_{0}}{\left(\rho C \pi r_{0}^{2}\right)^{2}} \frac{1}{\omega^{2}}
$$

For the exact calculations for half-space (see Appendix A) we introduce the displacement $\bar{X}$ averaged over the beam spot on the surface

$$
\bar{X}=\frac{1}{\pi r_{0}^{2}} \iint_{-\infty}^{\infty} d y d z, v_{x}(x=0, y, z) e^{-\left(y^{2}+z^{2}\right) / r_{0}^{2}}
$$

where $v_{x}(x=0, y, z)$ is $x$-component of vector of deformation on the surface, and find the spectral density of $\bar{X}$ :

$$
S_{\mathrm{TS}}(\omega)=2 \alpha^{2}(1+\sigma)^{2} \frac{\hbar \omega_{0} W_{0}}{\left(\rho C \pi r_{0}^{2}\right)^{2}} \frac{1}{\omega^{2}}
$$

where $\sigma$ is the Poisson coefficient.

${ }^{1}$ We use "one-sided" spectral density, defined only for positive frequencies, which may be calculated from correlation function $\langle X(t) X(t+\tau)\rangle$ using formula

$$
S_{X}(\omega)=2 \int_{-\infty}^{\infty} d \tau\langle X(t) X(t+\tau)\rangle \cos (\omega \tau)
$$


Here and below we use the fact that for materials at room temperature the thermal relaxation time of the spot $\tau_{T} \simeq r_{0}^{2} / a^{2}$ is very large:

$$
\omega \gg \frac{a^{2}}{r_{0}^{2}}
$$

It is easy to see that the exact solution (8) differs from the approximate one (6) only by Poisson coefficient.

\section{THERMODYNAMICAL FLUCTUATIONS OF TEMPERATURE, THERMOELASTIC DAMPING AND SURFACE FLUCTUATIONS}

It is known from thermodynamics [14] that the total variance of fluctuations of temperature in volume $V$ is described by the following simple formula:

$$
\left\langle\delta T^{2}\right\rangle=\frac{\kappa T^{2}}{\rho C V}
$$

where $\kappa$ is the Boltzmann constant. In classical solid state thermodynamics these fluctuations are not correlated with fluctuations of volume (which are responsible for the Brownian

noise) [14 but this is not the case for nonzero thermal expansion coefficient. To find the influence of the fraction of this type of fluctuations in the vicinity of $\omega_{\text {grav }}$ on the vibration of the surface along the $\mathrm{x}$-axis in direct way we use the Langevin approach and introduce fluctuational thermal sources $F(\vec{r}, t)$ added to the right part of the equation of thermal conductivity:

$$
\frac{\partial u}{\partial t}-a^{2} \Delta u=F(\vec{r}, t)
$$

These sources should be normalized in a way to satisfy formula (10). By substituting the solution of this equation for the temperature $u$ into the equation of elasticity and averaging displacement (7) over the spot on the surface, we find spectral density of its fluctuations in frequency range of interest (see details in Appendix B):

$$
S_{\mathrm{TD}}(\omega) \simeq \frac{8}{\sqrt{2 \pi}} \alpha^{2}(1+\sigma)^{2} \frac{\kappa T^{2}}{\rho C} \frac{a^{2}}{r_{0}^{3}} \frac{1}{\omega^{2}} \quad \text { if } \quad \frac{r_{0}^{2} \omega}{a^{2}} \gg 1 .
$$


We can also find the spectral density of displacement using Fluctuation-Dissipation theorem (FDT) if we assume that the only dissipation mechanism in the mirror is thermoelastic damping. Elastic deformations in a solid body through thermal expansion lead to inhomogeneous distribution of temperature and hence to fluxes of heat and losses of energy [19. The same approach was used [17, 18] for the calculation of vibration of the surface due to the Brownian fluctuations. To calculate the fluctuations in this approach we should apply a periodic pressure $p$ distributed over the beam spot on the surface:

$$
p(y, z, t)=\frac{F_{0}}{\pi r_{0}^{2}} e^{-\left(y^{2}+z^{2}\right) / r_{0}^{2}} e^{i \omega t}
$$

and we should find susceptibility $\chi=\bar{X} / F_{0}$. After that in accordance with FDT the spectral density of displacement $\bar{X}$ must be proportional to imaginary part of susceptibility $\chi$ :

$$
S_{x}(\omega)=\frac{4 \kappa T}{\omega}|\operatorname{Im}(\chi(\omega))|
$$

Calculations (see Appendix C) show that FDT gives the same result as formula (12). Therefore, we can conclude that thermodynamical fluctuations of temperature are the physical source of fluctuations deduced from FDT based on thermoelastic damping.

Calculations of $\operatorname{Im}(\chi)$ are rather bulky but Zener [19] suggested a very simple approach allowing to estimate thermoelastic loss angles and hence the imaginary parts of elastic coefficients. It allows to find $\operatorname{Im}(\chi)$ only from formula for $\chi$ in zero order approximation (see Appendix C):

$$
\chi^{(0)}(\omega)=\frac{\left(1-\sigma^{2}\right)}{\sqrt{2 \pi} E r_{0}}
$$

where $E$ is the Young's modulus.

The imaginary parts of elastic coefficients due to thermoelastic damping may be calculated in Zener's approach according to the formulas [15]:

$$
\begin{array}{r}
\frac{\operatorname{Im}(\sigma)}{\sigma}=\frac{\sigma_{S}-\sigma_{T}}{\sigma_{T}} \frac{\omega \tau_{T}}{1+\omega^{2} \tau_{T}^{2}}=\frac{\alpha^{2} T E}{C \rho} \frac{1+\sigma}{\sigma} \frac{\omega \tau_{T}}{1+\omega^{2} \tau_{T}^{2}}, \\
\quad \frac{\operatorname{Im}(E)}{E}=\frac{E_{S}-E_{T}}{E_{T}} \frac{\omega \tau_{T}}{1+\omega^{2} \tau_{T}^{2}}=\frac{\alpha^{2} T E}{C \rho} \frac{\omega \tau_{T}}{1+\omega^{2} \tau_{T}^{2}}
\end{array}
$$


Where $E_{T}, E_{S}$ are consequently iso-thermic and adiabatic Young's moduluses $\left(E_{T} \simeq E_{S} \simeq\right.$ $E)$ and $\sigma_{T}, \sigma_{S}$ are iso-thermic and adiabatic Poisson coefficients $\left(\sigma_{T} \simeq \sigma_{S} \simeq \sigma\right), \tau_{T}$ is the time of thermal relaxation of our spot. By substituting these values in (15) and then in (14) and taking into account that $\tau_{T}=2 r_{0}^{2} / a^{2} \gg 1 / \omega$ (this relaxation time may be found relatively easy from the solution of the problem of thermal relaxation of the heated spot) we finally obtain the same formula (12).

It is really worth noting that all the three methods give precisely the same result.

\section{NUMERICAL ESTIMATES}

Now we want to compare the fluctuations of the mirrors' surface caused by the described above mechanisms with other known sources of noise in LIGO antennae.

The main determining source of noise associated with the mirrors' material is usually described by the losses in the model of structural damping (we denote it as Brownian motion of the surface). In this model the angle of losses $\phi$ does not depend on frequency and analogously to 8, 17, 18 by substituting $E=\bar{E}(1+i \phi)$ in (15) and then in (14) we obtain:

$$
S_{\mathrm{SD}}(\omega) \simeq \frac{4 \kappa T}{\omega} \frac{\left(1-\sigma^{2}\right)}{\sqrt{2 \pi} E r_{o}} \phi
$$

For simplicity here we neglected the unknown imaginary part of Poisson coefficient.

The sensitivity of gravitational wave antenna to the perturbation of metric may be recalculated from noise spectral density of displacement $X$ using the following formula:

$$
h(\omega)=\frac{\sqrt{2 S_{X}}}{L}
$$

where we used the fact that antenna has two arms (with length $L$ ) and in each arm only one mirror adds to noise (on end mirrors the fluctuations are averaged over the larger beam spot).

The LIGO-II antenna will reach the level of SQL, so we also compare the noise limited sensitivity to this limit in spectral form [12]: 


$$
h(\omega)=\sqrt{\frac{8 \hbar}{m \omega^{2} L^{2}}} .
$$

On figures 1 and 2 we plot the sensitivity limitations for the perturbation of metric caused by the described thermal mechanisms and SQL (formulas 8, 12, 17, 18, 19) for fused silica (Fig.1) and sapphire (Fig.2) mirrors. We used the following numerical values for the parameters of the two materials - fused silica and sapphire. (We should note that the actual figures, which various sapphire manufactures provide, significantly vary - tens of percents, especially for the thermal expansion coefficient):

$$
\begin{aligned}
& \omega=2 \pi \times 100 \mathrm{~s}^{-1}, \quad r_{0}=1.56 \mathrm{~cm}, \quad T=300 \mathrm{~K}, \\
& \omega_{0}=2 \times 10^{15} \mathrm{~s}^{-1}, \quad L=4 \times 10^{5} \mathrm{~cm}, \quad W_{0}=10^{7} \mathrm{erg} / \mathrm{s} ; \\
& \text { Fused silica: } \quad \alpha=5.5 \times 10^{-7} \mathrm{~K}^{-1}, \quad \lambda^{*}=1.4 \times 10^{5} \frac{\mathrm{erg}}{\mathrm{cm} \mathrm{s} \mathrm{K}}, \\
& \rho=2.2 \frac{\mathrm{g}}{\mathrm{cm}^{3}}, \quad C=6.7 \times 10^{6} \frac{\mathrm{erg}}{\mathrm{g} \mathrm{K}}, \quad m=1.1 \times 10^{4} \mathrm{~g}, \\
& E=7.2 \times 10^{11} \frac{\mathrm{erg}}{\mathrm{cm}^{3}}, \quad \sigma=0.17, \quad \phi=5 \times 10^{-8} \\
& \text { Sapphire: } \quad \alpha=5.0 \times 10^{-6} \mathrm{~K}^{-1}, \quad \lambda^{*}=4.0 \times 10^{6} \frac{\mathrm{erg}}{\mathrm{cm} \mathrm{s} \mathrm{K}}, \\
& \rho=4.0 \frac{\mathrm{g}}{\mathrm{cm}^{3}}, \quad C=7.9 \times 10^{6} \frac{\mathrm{erg}}{\mathrm{g} \mathrm{K}}, \quad m=3 \times 10^{4} \mathrm{~g}, \\
& E=4 \times 10^{12} \frac{\mathrm{erg}}{\mathrm{cm}^{3}}, \quad \sigma=0.29, \quad \phi=3 \times 10^{-9} .
\end{aligned}
$$

From these two figures it is evident that the effects associated with thermal expansion in no case may be neglected. Especially unfortunate result is obtained for sapphire - the noise from thermodynamical fluctuations of temperature dominates for frequencies of interest and is several times larger than the SQL. While for fused silica the situation is the opposite. The SQL is one order larger than TD fluctuations. It is important to note, that unlike structural damping for which angles of losses at low frequencies were not yet directly measured, our mechanism has fundamental nature and is calculated explicitely without model assumptions. 


\section{CONCLUSION}

The analysis and numerical estimates presented above for two effects allow to give some recommendations for the strategy of upgrading laser interferometric gravitational wave antennae.

1. It is important to emphasize that contrastingly to the gravitational wave, both effects do not displace the centers of masses of the mirrors. Thus, in principle it is possible to subtract these effects as well as usual Brownian fluctuations (with certain level of accuracy) in a way similar to the procedure suggested for the subtraction of thermal noise in suspension fibers [20]. However, till now to our knowledge nobody presented the scheme of such subtraction.

2. Potentially there exists a possibility to decrease substantially these effects by choosing materials for the mirrors with substantially smaller values of $\alpha$. There also exists the possibility to use special cuts of anisotropic monocrystals for which $\alpha$ turns to zero.

3. Thermodynamic fluctuations decreese with growing radius of the beam spot as $r_{0}^{3 / 2}$ even stronger then for the Brownian fluctuations. In this way, some optimization of the geometry of the resonators may help.

4. The "brute force" method for the improvement of the sensitivity by increasing the circulating optical power $W$ does not look promising, taking into account photo-thermal shot noise. It seems that few mega-Watts is the upper limit for $W$.

\section{ADDENDUM}

The evident method to suppress the thermodynamical fluctuations by using larger beamspots is unfortunately limited by diffraction losses and technology. If we choose the LIGO-I geometry with beam sizes on internal and end mirrors: $r_{0 i}=3.6 / \sqrt{2} \mathrm{~cm}, \quad r_{0 e}=$ $4.6 / \sqrt{2} \mathrm{~cm}$ then the threshold of sensitivity of thermodynamical fluctuations will be only 1.7 times lower than on our graphics still 2 times higher than the SQL. 


\section{ACKNOWLEDGMENTS}

We are pleased to thank for helpful discussions V.P.Mitrofanov, F.Ya.Khalili, A.I.Osipov, I.A.Kvasnikov, H.-J.Kimble and R.Weiss. This research was partially supported by the California Institute of Technology, US National Science Foundation and the Russian Foundation for Basic Research grant \#96-15-96780.

\section{APPENDIX A.}

In this Appendix we calculate the spectral density (8) of the fluctuations of the averaged displacement $\bar{X}$ caused by photo-thermal shot noise.

We start from thermal conductivity equation:

$$
\begin{aligned}
& \frac{\partial u}{\partial t}-a^{2} \Delta u=\frac{w(t)}{\rho C} \frac{1}{\pi r_{0}^{2}} 2 \delta(x) \exp \left(-\frac{y^{2}+z^{2}}{r_{0}^{2}}\right) \\
& \left\langle w(t) w\left(t^{\prime}\right)\right\rangle=\hbar \omega_{0} W_{0} \delta\left(t-t^{\prime}\right), \quad w(t)=W_{\text {abs }}(t)-W_{0}, \quad W_{0}=\left\langle W_{\text {abs }}\right\rangle,
\end{aligned}
$$

with boundary condition (2) and equation of elasticity for the field of deformation $\vec{v}(x, y, z)$ (see [15,16]) with boundary conditions for tensions on free surface:

$$
\begin{aligned}
& \frac{1-\sigma}{1+\sigma} \operatorname{grad} \operatorname{div} \vec{v}-\frac{1-2 \sigma}{2(1+\sigma)} \operatorname{rot} \operatorname{rot} \vec{v}=\alpha \operatorname{grad} u \\
& \sigma_{x x}=\left.\frac{E}{1-2 \sigma}\left[\frac{\sigma}{1+\sigma}\left(\frac{\partial v_{x}}{\partial x}+\frac{\partial v_{y}}{\partial y}+\frac{\partial v_{z}}{\partial z}\right)-\alpha u+\frac{1-2 \sigma}{1+\sigma} \frac{\partial v_{x}}{\partial x}\right]\right|_{x=0}=0 \\
& \sigma_{x y}=\left.\frac{E}{2(1+\sigma)}\left(\frac{\partial v_{x}}{\partial y}+\frac{\partial v_{y}}{\partial x}\right)\right|_{x=0}=0, \quad \sigma_{x z}=\left.\frac{E}{2(1+\sigma)}\left(\frac{\partial v_{x}}{\partial z}+\frac{\partial v_{z}}{\partial x}\right)\right|_{x=0}=0
\end{aligned}
$$

The problem (24, 2) in half-space can be replaced by the problem (24) in full space, in which the function of source is evenly continued for negative $x$. In this case the condition (2) is satisfied automatically. Assuming that the absorbed power changes as $w(t)=w(\omega) e^{i \omega t}$ one can find the spectral component:

$$
u(\vec{r}, \omega)=\iiint_{-\infty}^{\infty} \frac{d k_{x} d k_{y} d k_{z}}{(2 \pi)^{3}} e^{i k_{x} x+i k_{y} y+i k_{z} z} e^{-r_{0}^{2}\left(k_{y}^{2}+k_{z}^{2}\right) / 4} \frac{2 w(\omega)}{\rho C\left(a^{2} k^{2}+i \omega\right)} .
$$

After substitution of $u(\vec{r}, \omega)$ into (25, 26) one can find the solution for $\vec{v}$ as a sum [16] $\vec{v}=\operatorname{grad} \varphi+\vec{v}^{(a)}$, where $\varphi$ satisfies Poisson's equation (it can be deduced from (25)): 


$$
\Delta \varphi=\frac{1+\sigma}{1-\sigma} \alpha u
$$

without boundary conditions and function $\vec{v}^{(a)}$ satisfies equation (25) with zero right part and boundary conditions:

$$
\begin{aligned}
& \sigma_{x x}=\left.\frac{E}{1+\sigma}\left[\frac{\partial^{2} \varphi}{\partial y^{2}}+\frac{\partial^{2} \varphi}{\partial y^{z}}\right]\right|_{x=0}, \\
& \sigma_{x y}=-\left.\frac{E}{(1+\sigma)} \frac{\partial^{2} \varphi}{\partial x \partial y}\right|_{x=0}, \quad \sigma_{x z}=-\left.\frac{E}{(1+\sigma)} \frac{\partial^{2} \varphi}{\partial x \partial z}\right|_{x=0} .
\end{aligned}
$$

It is convenient to choose the function $\varphi$ in symmetrical form (by evenly continuing the $u(\vec{r}, \omega)$ for negative values of $x)$ :

$$
\begin{gathered}
\varphi(x, y, z, \omega)=-\frac{\alpha(1+\sigma)}{4 \pi(1-\sigma)} \iiint_{-\infty}^{\infty} \frac{d x^{\prime} d y^{\prime} d z^{\prime} u(\vec{r}, \omega)}{\sqrt{\left(x^{\prime}\right)^{2}+\left(y-y^{\prime}\right)^{2}+\left(z-z^{\prime}\right)^{2}}} \\
\varphi(x=0, y, z, \omega)=-\frac{\alpha(1+\sigma)}{(1-\sigma)} \frac{2 w(\omega)}{\rho C} \iiint_{-\infty}^{\infty} \frac{d k_{x} d k_{y} d k_{z}}{(2 \pi)^{3} k^{2}\left(a^{2} k^{2}+i \omega\right)} e^{-r_{0}^{2}\left(k_{y}^{2}+k_{z}^{2}\right) / 4+i k_{y} y+i k_{z} z} .
\end{gathered}
$$

In this case $\partial_{x} \varphi(x=0, y, z)=0$ and therefore (i) the function $\varphi$ gives no contribution into $\bar{X}$ and (ii) tangential tensions $\sigma_{x y}$ and $\sigma_{x z}$ in boundary conditions (29) turn to zero.

The solution of the problem for $\vec{v}^{(a)}$ is known (see for example [15]). Using condition (9) and following table integrals:

$$
\begin{gathered}
\iint_{-\infty}^{\infty} d y d z \frac{e^{i k_{y} y+i k_{z} z}}{\sqrt{y^{2}+z^{2}}}=\frac{2 \pi}{k_{\perp}}, \quad k_{\perp}=\sqrt{k_{y}^{2}+k_{z}^{2}} \\
\iiint_{-\infty}^{\infty} d x d y d z \frac{e^{i k_{x} x+i k_{y} y+i k_{z} z}}{\sqrt{x^{2}+y^{2}+z^{2}}}=\frac{4 \pi}{k^{2}}, \quad k=\sqrt{k_{x}^{2}+k_{y}^{2}+k_{z}^{2}}
\end{gathered}
$$

we substitute $\vec{v}^{(a)}$ into (7) and obtain:

$$
\begin{aligned}
\bar{X}(\omega) & =-4 \alpha(1+\sigma) \frac{w(\omega)}{\rho C} \iiint_{\infty}^{\infty} \frac{d k_{x} d k_{y} d k_{z}}{(2 \pi)^{3}} \frac{k_{\perp}}{k^{2}\left(a^{2} k^{2}+i \omega\right)} e^{-r_{0}^{2}\left(k_{y}^{2}+k_{z}^{2}\right) / 2} \simeq \\
& \simeq-\frac{\alpha(1+\sigma)}{i \pi^{2} \omega} \frac{w(\omega)}{\rho C} \int_{-\infty}^{\infty} d k_{x} \int_{0}^{\infty} d k_{\perp} \frac{k_{\perp}}{k_{x}^{2}+k_{\perp}^{2}} e^{-r_{0}^{2} k_{\perp}^{2} / 2}=-\frac{\alpha(1+\sigma)}{i \pi} \frac{w(\omega)}{\rho C r_{0}^{2}} \frac{1}{\omega}
\end{aligned}
$$

Assuming that spectral density of absorbed power is equal to $S_{w}(\omega)=2 \hbar \omega_{0} W_{0}$ one can find the formula (8). 


\section{APPENDIX B}

In this Appendix we deduce the formula (12) using Langevin abroach for thermal conductivity equation (11) and recalculate the thermo-dynamical fluctuations of temperature into deformations.

Using time and space Fourier transform one can write down the formal solution of (11):

$$
u(\vec{r}, t)=\iint_{-\infty}^{\infty} \frac{d \vec{k} d \omega}{(2 \pi)^{4}} \frac{F(\vec{k}, \omega)}{a^{2}(\vec{k})^{2}+i \omega} e^{i \omega t+i \vec{k} \vec{r}},
$$

We interest in temperature $\bar{u}$ averaged over some volume $V$. Assuming that

$$
\begin{aligned}
& \left\langle F(\vec{k}, \omega) F^{*}\left(\vec{k}^{\prime}, \omega^{\prime}\right)\right\rangle=(2 \pi)^{4} F_{0}^{2}|\vec{k}|^{2} \delta\left(\vec{k}-\vec{k}^{\prime}\right) \delta\left(\omega-\omega^{\prime}\right) \text { one can calculate }\left\langle\bar{u}^{2}\right\rangle \\
& \left\langle\vec{u}^{2}\right\rangle=\frac{1}{V} \int_{V} d \vec{r} \frac{1}{V} \int_{V} d \vec{r}_{1} \iiint \int_{-\infty}^{\infty} \frac{d \vec{k} d \vec{k}^{\prime} d \omega d \omega^{\prime}}{(2 \pi)^{8}} \frac{\left\langle F(\vec{k}, \omega) F^{*}\left(\vec{k}^{\prime}, \omega^{\prime}\right)\right\rangle}{\left(a^{2}(\vec{k})^{2}+i \omega\right)\left(a^{2}\left(\vec{k}^{\prime}\right)^{2}+i \omega^{\prime}\right)} e^{i\left(\omega-\omega^{\prime}\right) t+i \vec{k} \vec{r}-i \vec{k}^{\prime} \vec{r}_{1}}= \\
& \quad=\frac{F_{0}^{2}}{2 a^{2}} \frac{1}{V}, \quad \rightarrow \quad F_{0}^{2}=2 a^{2} \frac{\kappa T}{\rho C}
\end{aligned}
$$

Here we equate (35) to (10) and find $F_{0}^{2}$.

In order to find TD temperature fluctuations in half-space with boundary condition (2) one can solve equivalent problem for full space with source function even continued for negative $x$, so that its correlator is equal to:

$$
\left\langle F(\vec{k}, \omega) F^{*}\left(\vec{k}^{\prime}, \omega^{\prime}\right)\right\rangle=(2 \pi)^{4} F_{0}^{2}|\vec{k}|^{2}\left[\delta\left(k_{x}-k_{x}^{\prime}\right)+\delta\left(k_{x}+k_{x}^{\prime}\right)\right] \delta\left(k_{y}-k_{y}^{\prime}\right) \delta\left(k_{z}-k_{z}^{\prime}\right) \delta\left(\omega-\omega^{\prime}\right)
$$

After that we must substitute the expression (34) for $u$ into elasticity equation (25, 26). The solution for $\vec{v}$ can be found according to the same scheme as in Appendix A: $\vec{v}=\operatorname{grad} \varphi+\vec{v}^{(a)}$, where $\varphi$ satisfies Poisson equation (28). Again $\varphi$ can be written in symmetrical form (30) and the function $\vec{v}^{(a)}$ must satisfy equation (25) with zero right part and following boundary conditions:

$$
\begin{aligned}
& \sigma_{x x}(x=0, y, z)=\frac{\alpha E}{(1-\sigma)} \iiint \int_{-\infty}^{\infty} \frac{d k_{x} d k_{y} d k_{z} d \omega}{(2 \pi)^{4}} \frac{F(\vec{k}, \omega)}{a^{2} k^{2}+i \omega} \frac{k_{y}^{2}+k_{z}^{2}}{k^{2}} e^{i \omega t+i k_{y} y+i k_{z} z}, \\
& \sigma_{x y}(x=0, y, z)=\sigma_{x z}(x=0, y, z)=0 .
\end{aligned}
$$

The solution for $\vec{v}^{(a)}$ is known [15]

$$
v_{x}^{(a)}(x=0, y, z)=\frac{1-\sigma^{2}}{\pi E} \iint_{-\infty}^{\infty} d y^{\prime} d z^{\prime} \frac{\sigma_{x x}\left(x=0, y^{\prime}, z^{\prime}\right)}{\sqrt{\left(y-y^{\prime}\right)^{2}+\left(z-z^{\prime}\right)^{2}}} .
$$


Substituting it into (7) we find $\bar{X}(t)$ and spectral density $S_{T D}(\omega)$ :

$$
\begin{aligned}
\bar{X}(t) & =2 \alpha(1+\sigma) \iiint \int_{-\infty}^{\infty} \frac{d k_{x} d k_{y} d k_{z} d \omega}{(2 \pi)^{4}} \frac{F(\vec{k}, \omega)}{a^{2} k^{2}+i \omega} \frac{k_{\perp}}{k^{2}} e^{i \omega t-k_{\perp}^{2} r_{0}^{2} / 4}, \\
S_{T D}(\omega) & =8 \alpha^{2}(1+\sigma)^{2} \iiint_{-\infty}^{\infty} \frac{d k_{x} d k_{y} d k_{z}}{(2 \pi)^{3}} \frac{F_{0}^{2}}{a^{4} k^{4}+\omega^{2}} \frac{2\left(k_{\perp}^{2}\right)}{k^{2}} e^{-k_{\perp}^{2} r_{0}^{2} / 2}
\end{aligned}
$$

From the last formula, using integration in spherical coordinate system and neglecting the term $a^{4} k^{4}$ in denominator according to condition (9), we obtain formula (12) asymptotically correct for the frequencies $\omega r_{0}^{2} / a^{2} \gg 1$.

The table integrals (31, 32) was used above in calculations of $\sigma_{x x}(x=0, y, z), v_{x}^{(a)}(x=$ $0, y, z), \bar{X}(t)$.

\section{APPENDIX C}

To calculate the fluctuations of the surface using FDT approach with thermoelastic losses we should solve the system:

$$
\begin{aligned}
& \frac{1-\sigma}{1+\sigma} \operatorname{grad} \operatorname{div} \vec{v}-\frac{1-2 \sigma}{2(1+\sigma)} \operatorname{rot} \operatorname{rot} \vec{v}=\alpha \operatorname{grad} u, \\
& \frac{\partial u}{\partial t}-a^{2} \Delta u=\frac{\alpha E T}{C \rho(1-2 \sigma)} \frac{\partial \operatorname{div} \vec{v}}{\partial t}, \\
& \sigma_{x x}=\frac{F_{0}}{\pi r_{0}^{2}} e^{-\left(y^{2}+z^{2}\right) / r_{0}^{2}} \quad \sigma_{x y}=\sigma_{x z}=\left.0 \quad \frac{\partial u}{\partial x}\right|_{x=0}=0 .
\end{aligned}
$$

In zero order approximation we simply solve elasticity problem neglecting temperature $u$ in the right part of the first equation in (36) and in boarder conditions. This solution is well known [15]:

$$
\begin{aligned}
& \vec{v}=\frac{F_{0}(1+\sigma)}{2 \pi^{2} E r_{0}^{2}} \iint_{-\infty}^{\infty} e^{-\left(y^{\prime 2}+z^{\prime 2}\right) / r_{0}^{2}} \vec{G}\left(\vec{r}-\vec{r}^{\prime}\right) d y^{\prime} d z^{\prime} \\
& \vec{G}(\vec{r})=\left(\frac{2(1-\sigma)}{r}+\frac{x^{2}}{r^{3}}\right) \vec{e}_{x}+\left(\frac{x}{r^{3}}-\frac{1-2 \sigma}{r(r+x)}\right)\left(y \vec{e}_{y}+z \vec{e}_{z}\right)
\end{aligned}
$$

By substituting this solution in (7) and using (31) we obtain susceptibility $\chi^{(0)}$ (15). This

result totally coincides with the calculations for the half-space in [18] (if we note that the stored static strain energy $U=\bar{X}^{(0)} F_{0} / 2=F_{0}^{2} \chi^{(0)} / 2$ and $\left.w_{0}=\sqrt{2} r_{0}\right)$ and has minor 
numerical difference with the approximation in [17. To find first order approximation for the distribution of temperature we substitute

$$
\operatorname{div} \vec{v}^{(0)}=-\frac{(1+\sigma)(1-2 \sigma) F_{0}}{2 \pi^{2} E} \iint_{-\infty}^{\infty} e^{-k_{\perp}^{2} r_{0}^{2} / 4-k_{\perp} x+i k_{y} y+i k_{z} z} d k_{y} d k_{z}
$$

in (37) to find that

$$
u^{(1)}(\vec{r}, t)=\frac{4 \alpha T(1+\sigma) F_{0} i \omega}{C \rho} \iiint_{-\infty}^{\infty} \frac{d k_{x} d k_{y} d k_{z}}{(2 \pi)^{3}} \frac{k_{\perp}}{k^{2}\left(a^{2} k^{2}+i \omega\right)} e^{-k_{\perp}^{2} r_{0}^{2} / 4+i \vec{k} \vec{r}} .
$$

We then substitute this solution in (36) to find second order approximation in the same way as in the two Appendices above by finding appropriate evenly continued $\varphi$ function satisfying (28, 29). We find using (32)

$$
\sigma_{x x}(0, y, z)=-\frac{4(1+\sigma) \alpha^{2} T E F_{0} i \omega}{(1-\sigma) \rho C} \iiint_{-\infty}^{\infty} \frac{d k_{x} d k_{y} d k_{z}}{(2 \pi)^{3}} \frac{k_{\perp}^{3}}{k^{4}\left(a^{2} k^{2}+i \omega\right)} e^{-k_{\perp}^{2} r_{0}^{2} / 4+i k_{y} y+i k_{z} z} .
$$

By substituting this expression in

$$
v_{x}^{(2 a)}(0, y, z)=\frac{1-\sigma^{2}}{\pi E} \iint_{-\infty}^{\infty} d y^{\prime} d z^{\prime} \frac{\sigma_{x x}\left(0, y^{\prime}, z^{\prime}\right)}{\sqrt{\left(y-y^{\prime}\right)^{2}+\left(z-z^{\prime}\right)^{2}}}
$$

and using (7) we may finally obtain

$$
X^{(2)}=-8(1+\sigma)^{2} \frac{\alpha^{2} T}{\rho C} F_{0} i \omega \iiint_{-\infty}^{\infty} \frac{d k_{x} d k_{y} d k_{z}}{(2 \pi)^{3}} \frac{k_{\perp}^{2}}{k^{4}\left(a^{2} k^{2}+i \omega\right)} e^{-k_{\perp}^{2} r_{0}^{2} / 2}
$$

and hence $\operatorname{Im}(\chi(\omega))$ which leads through (14) to precisely the same expression as (12). 


\section{REFERENCES}

[1] A. Abramovici et al., Science 256(1992)325.

[2] A. Abramovici et al., Phys.Letters.A 218, 157 (1996).

[3] I. Batov, M. Dantchevskaya, Proc. of VII All-Russian conf. on silica glasses, St.Petersburg 1991, p.45.

[4] A. Gillespi and F. Raab, Phys. Rev. D 52, 577 (1995)

[5] V.B. Braginsky, V.P. Mitrofanov and V.I. Panov, Systems with small dissipations, ed. by K.S. Thorne, Chicago Univ. Press (1985).

[6] V.B. Braginsky, V.P. Mitrofanov and K.V. Tokmakov, Phys.Letts.A 218, 164 (1996).

[7] S. Rowan, S. Twyford, R. Hutchins, J. Kovalik, J.E. Logan, A.C. McLaren, N.A. Robertson, J. Hough, Phys.Letts.A, 233, 303 (1997).

[8] P.R. Saulson, Rhys. Rev. D, 42, 2437 (1990);

G.I. Gonzalez and P.R. Saulson, J. Acoust. Soc. Am., 96, 207 (1994).

[9] A.Yu. Ageev, I.A. Bilenko, V.B. Braginsky Phys.Letts.A, 246, 479-484 (1998).

[10] V.B. Braginsky and S.P. Vyatchanin, Sov. Phys. JETP, 47, 433 (1978).

[11] J.H. Kimble, R. Weiss — private communication.

[12] V.B. Braginsky, M.L, Gorodetsky, F.Ya. Khalili and K.S. Thorne, Report at Third Amaldi Conference, Caltech, July, 1999, lanl e-print gr-qc/9907057.

[13] R.P. Jurchak, "Fizika zemli", \#7, 16 (1980) (in Russian).

[14] L.D. Landau and E.M. Lifshitz, Statistical Physics, Pergamon Press, New York (1984).

[15] L.D. Landau and E.M. Lifshitz, Theory of Elasticity, Pergamon Press, New York (1986), sec. $6,8,31$. 
[16] B.A. Boley and J.H. Wiener, "Theory of thermal stress", NY, John Wiley and Sons Inc., 1960, ch. 8.

[17] Yu. Levin, Phys. Rev. D, 57, 659-663 (1998)

[18] F. Bondu, P. Hello, Jean-Yves Vinet, Physics Letters bf A 246, 227 (1998).

[19] C. Zener, em Elasticity and Anelasticity of Metals, Chicahgo, The univ. of Chicago Press (1948).

[20] V.B. Braginsky, Yu. Levin and S.P. Vyatchanin, Meas. Sci. Technol., 10, 598 (1999). 


\section{FIGURES}

Fused Silica $\left(\mathrm{SiO}_{2}\right)$

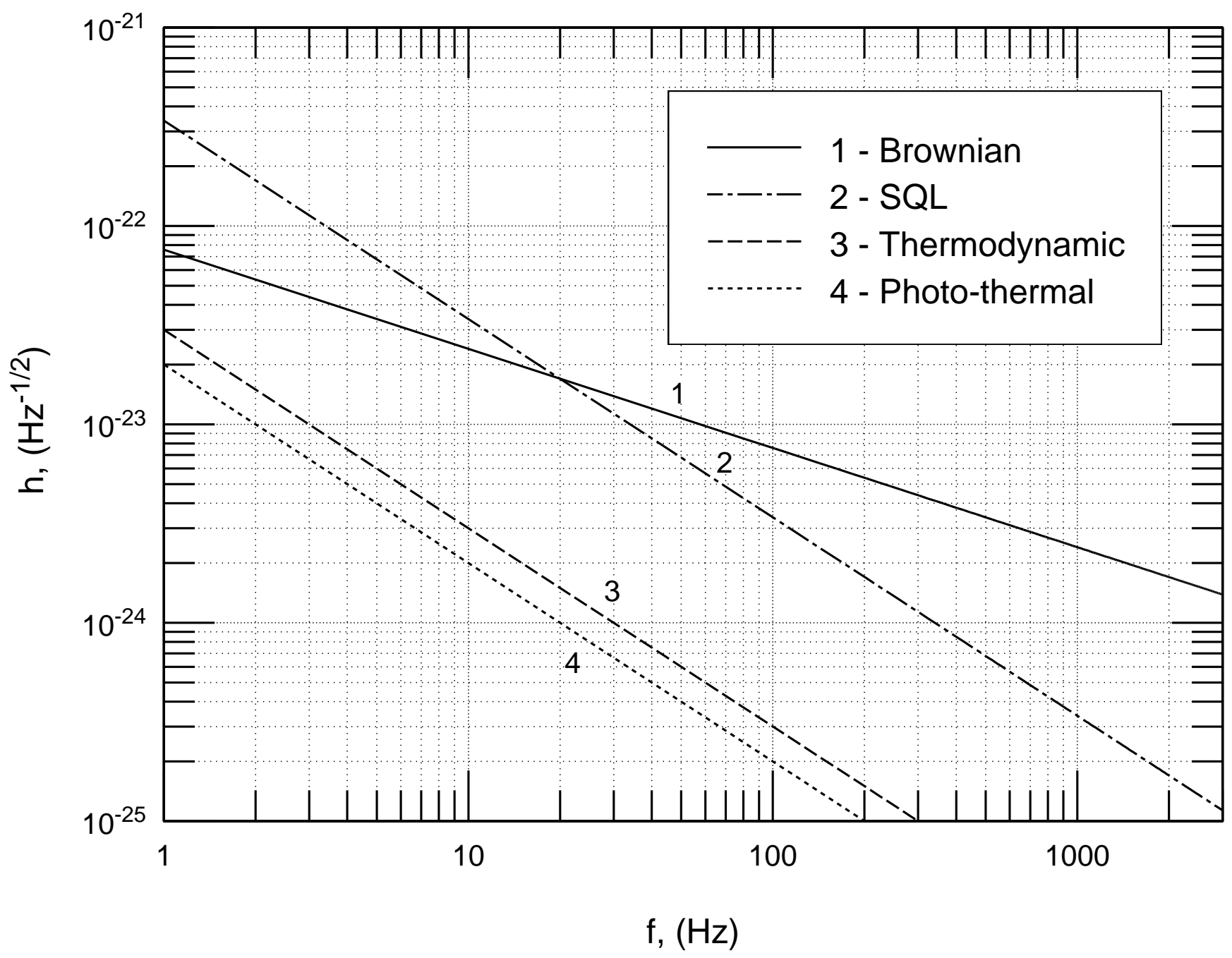




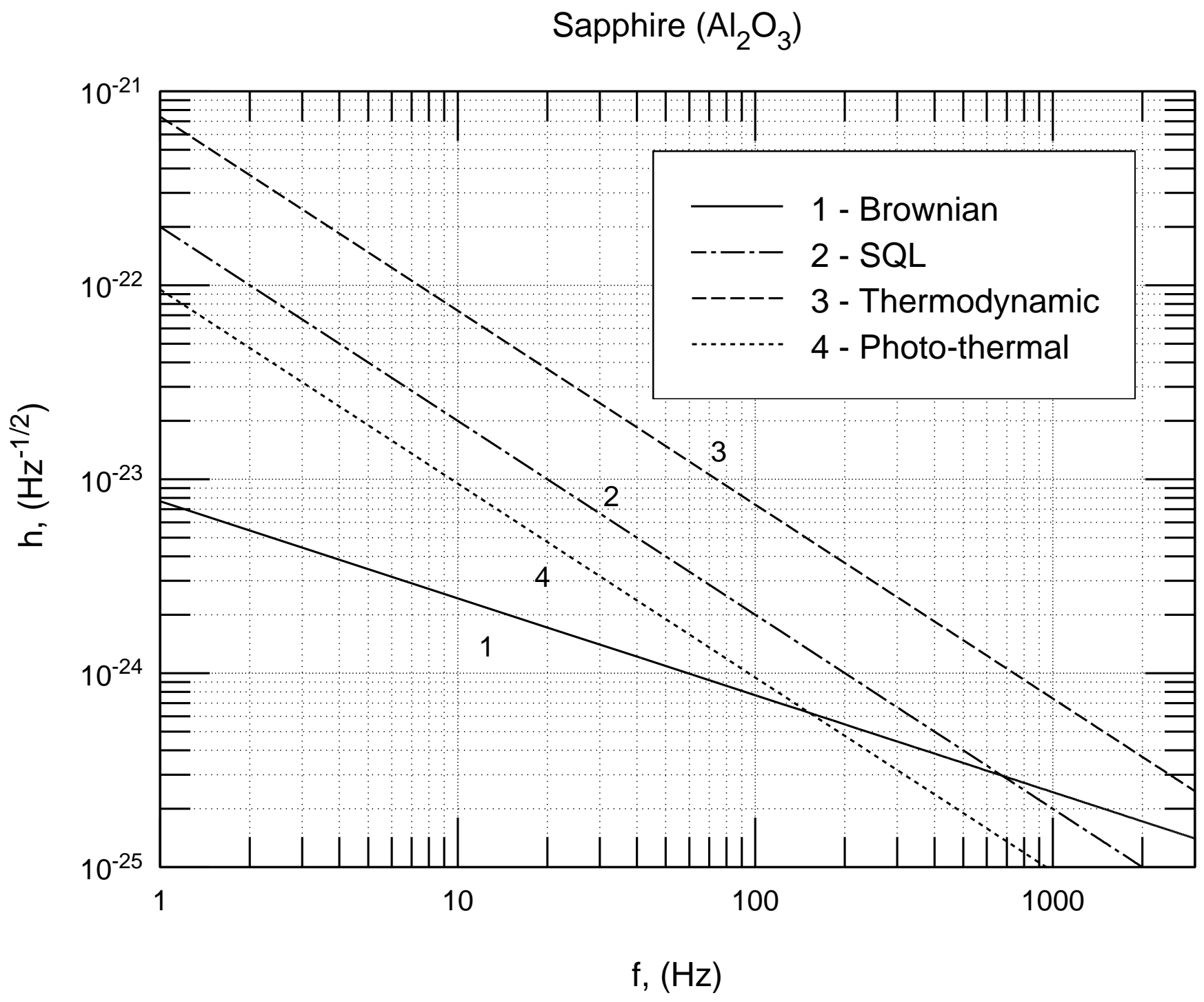


Figure captions:

Fig.1 The sensitivity limitations for the perturbation of metric caused by the SQL and by thermal fluctuations in fused silica mirrors.

Fig.2 The sensitivity limitations for the perturbation of metric caused by the SQL and by thermal fluctuations in sapphire mirrors. 